\title{
ON WAVEWISE ENTROPY INEQUALITIES FOR HIGH-RESOLUTION SCHEMES. I: THE SEMIDISCRETE CASE
}

\author{
HUANAN YANG
}

\begin{abstract}
We develop a new approach, the method of wavewise entropy inequalities for the numerical analysis of hyperbolic conservation laws. The method is based on a new extremum tracking theory and Vol'pert's theory of BV solutions. The method yields a sharp convergence criterion which is used to prove the convergence of generalized MUSCL schemes and a class of schemes using flux limiters previously discussed in 1984 by Sweby.
\end{abstract}

\section{INTRODUCTION}

In this paper, a new version of [29], we consider the entropy consistency of TVD and TVB high-resolution schemes for Cauchy problems of scalar conservation laws of the one space variable:

$$
\left\{\begin{array}{l}
w_{t}+f(w)_{x}=0 \\
w(x, 0)=w_{0}(x)
\end{array}\right.
$$

We assume that $f \in C^{2}$, and that $w_{0} \in B V$.

Let us partition the real line for the space variable into cells of equal size. The $j$ th cell is centered at $x_{j}=j h+c$, where $j=0, \pm 1, \pm 2, \ldots, c$ is a constant, and $h$ is the space stepsize. For an arbitrary function $v_{j}$ defined on the set of the grid points of the mesh, we use $\Delta_{+}$and $\Delta_{-}$to denote the forward and the backward difference operators, respectively: $\Delta_{ \pm} v_{j}= \pm\left(v_{j \pm 1}-v_{j}\right)$. The corresponding divided difference operators are denoted by $D_{ \pm}=\frac{1}{h} \Delta_{ \pm}$. Let $u_{j}(t)$ be the numerical approximation to the exact solution $w\left(x_{j}, t\right)$ or its cell average on the $j$ th cell $\bar{w}\left(x_{j}, t\right), t \leq \infty$. A semidiscrete conservative scheme has the form

$$
\frac{d}{d t} u_{j}(t)=-D_{+} g_{j-\frac{1}{2}}
$$

where

$$
g_{j+\frac{1}{2}}=g\left(u_{j-p+1}, u_{j-p+2}, \ldots, u_{j}, \ldots, u_{j+p}, h\right) .
$$

Here, $g$ is Lipschitz continuous with respect to its first $2 p$ arguments and is consistent with the conservation law in the sense that

$$
g(u, u, \ldots, u, h) \equiv f(u) .
$$

Received by the editor December 20, 1993 and, in revised form, September 13, 1994 and January 30, 1995.

1991 Mathematics Subject Classification. Primary 65M60, 65M12, 35L65.

Key words and phrases. Conservation law, MUSCL schemes, schemes using flux limiters, entropy condition, convergence. 
The collection of points $\left\{x_{j-p}, x_{j-p+1}, \ldots, x_{j+p}\right\}$ is said to be the stencil of the scheme at the point $\left(x_{j}, t\right)$, and the integer $p$ the size of the stencil of the scheme. The scheme is said to be self-similar if $g$ is independent of $h$.

We often extend the domain of a numerical solution $\left\{u_{j}(t): j=0, \pm 1, \pm 2, \ldots\right\}$ to the entire upper half plane $\mathbf{R} \times \mathbf{R}^{+}$by setting $u(x, t)=u_{j}(t)$ for $x_{j-1 / 2}<x<$ $x_{j+1 / 2}$.

Difficulties in regard to two important issues, nonlinear stability and entropy conditions, have hampered the advance in the analysis of high-order schemes. One can ease these difficulties with some stepsize-dependent limiters. For example, the works of Coquel and LeFloch [5], Johnson, Szepessy and Hansbo [12], Cockburn, Coquel and LeFloch [3], Cockburn and Gremaud [4], and Chen and Liu [2] all use stepsize-dependent limiters. These results are usually more general (multispace dimensions, nonconvex fluxes, systems, etc.). However, since these limiters smear discontinuities, especially the contact discontinuities (see [27] and [28]), one rarely uses them in computations.

Throughout this work, we only consider TVB self-similar schemes, and we abide by the following concept of convergence: A TVB scheme (1.2)-(1.4) for the Cauchy problem (1.1) converges if for each initial function $w_{0}$ in $\mathrm{BV}$, and for each sequence of initial data $\left\{u_{j}^{k}(0), j=0, \pm 1, \pm 2, \cdots\right\}_{k=1}^{\infty}$ such that they are uniformly in BV and converge in $L_{\text {loc }}^{1}(\mathbf{R})$ to $w_{0}$, and such that the stepsizes $h_{k} \rightarrow 0$ as $k \rightarrow \infty$, then the corresponding sequence of numerical solutions $\left\{u_{j}^{k}(t), j=0, \pm 1, \pm 2, \ldots\right\}_{k=1}^{\infty}$ of the scheme converges in $L_{\text {loc }}^{1}\left(\mathbf{R} \times \mathbf{R}^{+}\right)$to the unique entropy solution $w$ of the problem (1.1). By the Lax-Wendroff Theorem, entropy consistency implies convergence.

The dominant approach in the analysis of entropy consistency has been the method of cell entropy inequalities (CEI). In the works $[10,14,6,17,21]$, CEI was successfully used to prove the convergence of large classes of first-order schemes such as monotone schemes and E-schemes. Through the pioneering work [18] of Osher, in the last decade, CEI was further developed and applied to prove the convergence of a variety of high-resolution schemes for convex conservation laws. See also $[19,20,16]$ and the references therein.

The advantages of CEI are that the method mimics the elegant proof of the LaxWendroff Theorem, and that the method can be extended, at least in principle, to multi-dimensional cases and systems.

A disadvantage is that the method demands too much. For example, in the nonconvex case, the current uniqueness theory (see Vol'pert [26] and Kružkov [13]) requires the entropy inequalities for an entire class of entropy functions. The corresponding cell entropy inequalities exclude all but E-schemes which are only firstorder accurate (see [17] and [20]). Even in the convex case, this may necessitate unnatural restrictions (see [18]).

The recent interesting works of Bouchut, Bourdarias and Perthame [1], and Jiang and Shu [23] show that some self-similar schemes other than those in the form of (1.2)-(1.4) are more accommodating of cell entropy inequalities. However, the current paper will stick to the self-similar schemes in the form of (1.2)-(1.4), which contain most classical high-resolution schemes.

In this paper, we propose a new approach, the wavewise entropy inequalities (WEI), for a large class of TVD schemes. Roughly speaking, the WEI convergence criteria state that a scheme converges if it does not produce expansion shocks. As applications of the new approach, we solve two open problems concerning high- 
resolution schemes: We prove the convergence of generalized MUSCL schemes and the high-resolution schemes using Osher-Chakravarthy flux limiters.

In addition to providing sharp convergence criteria, the WEI rigorously describes and justifies a widely observed phenomenon: If a nonlinearly stable (say TVD) conservative scheme fails to converge, it produces expansion shocks. Moreover, the WEI provides a theoretical foundation for the extremely important practice of using Riemann (shock tube) problems as test problems for the numerical analysis of conservation laws.

The drawbacks of the WEI method are: 1. The method relies on TVD assumptions which rule out the possibility of applying its current version to some interesting self-similar uniformly high-order schemes. 2. Applications of the WEI method to high-resolution schemes for multi-dimensional conservation laws are even more remote. In this respect, according to the well-known Goodman-LeVeque Barrier Theorem (see [9]), two-dimensional TVD schemes are at most first-order accurate. To the author's knowledge, no self-similar scheme of higher than first-order accuracy has been proven to be TVB.

The paper is organized as follows. In $\S 2$ we review some properties of the discontinuities of BV weak solutions of conservation laws. In $\S 3$ we give four WEI convergence criteria and prove the first two and the last one of them. In $\S 4$ we present the applications. The proof of the third WEI criterion can be found in the Supplement to the paper, which contains the sections $\S 6$ to $\S 8$ and an appendix.

We point out here that when the first version of the IMA preprint [29] was completed by the end of 1989, Professor P. L. Lions kindly informed the author that in an independent work [private communication], with different techniques, he and P. E. Souganidis proved some convergence results for MUSCL schemes in the framework of Hamilton-Jacobi equations. It is understood that in one space dimension, entropy solutions of a hyperbolic conservation law correspond to viscosity solutions of the Hamilton-Jacobi equation with the same flux function. From their note [15], it is clear that, besides totally different techniques, the results of the two works for MUSCL schemes are also different: Our result is valid for any convex flux, for E-scheme building blocks, and for time-dependent problems; while the results in [15] are valid for strictly convex fluxes, for monotone-scheme building blocks, and for steady-state problems.

\section{REVIEW OF DisCONTINUITIES OF WEAK SOLUTIONS}

Unlike the method of CEI, the WEI method is a discrete version of the following simple observation concerning a weak solution $w$. Let $U(w)$ be an arbitrary convex entropy function, and let $F(w)$ be its entropy flux: $F^{\prime}(w)=U^{\prime}(w) f^{\prime}(w)$. If $w$ is smooth in an area $\Omega$, then $U_{t}+F_{x}=0$ holds there. Hence there is no need to worry about the entropy conditions at the area of smoothness of $w$ as long as it is a solution. In other words, the entropy consistency of a weak solution is solely determined by the entropy conditions of its discontinuities. In order to find the discrete version of this observation, we devote this section to a brief review of some of the key properties of these discontinuities. The main source for our review comes from Vol'pert's celebrated BV solution theory [26].

For completeness, we consider conservation laws $w_{t}+f(w)_{x}=0$ in several spatial dimensions. Hence, we assume that $x \in \mathbf{R}^{n}, f$ is a vector of $n$ components: $f=$ 
$\left(f_{1}, f_{2}, \ldots, f_{n}\right)^{T}$, and $f(w)_{x}=\sum \frac{\partial}{\partial x_{i}} f_{i}(w)$. We use $(a \bullet b)$ to denote the dot product of two vectors $a$ and $b$. Let $w_{-}$and $w_{+}$be any two distinct numbers in the domain of $f$. If $a$ is a unit vector that satisfies

$$
\left(w_{+}-w_{-}\right) a_{t}+\left(\left(f\left(w_{+}\right)-f\left(w_{-}\right)\right) \bullet a_{x}\right)=0,
$$

where $a_{t}$ and $a_{x}$ are the $t$-component and $x$-component of $a$, respectively, then the function

$$
W(x, t)= \begin{cases}w_{+} & \text {if }((x, t) \bullet a)>0, \\ w_{-} & \text {if }((x, t) \bullet a)<0\end{cases}
$$

is a traveling discontinuity which is a weak solution of the conservation law, and a is normal to the discontinuity. Define

$$
F(u, v)=[f(u)-f(v)] \operatorname{sgn}(u-v) .
$$

If $W(x, t)$ satisfies

$$
\left|w_{+}-c\right| a_{t}+\left(F\left(w_{+}, c\right) \bullet a_{x}\right) \leq\left|w_{-}-c\right| a_{t}+\left(F\left(w_{-}, c\right) \bullet a_{x}\right)
$$

for all real constants $c$, then $W$ is an admissible traveling discontinuity. Otherwise it is a traveling expansion shock. Let $f\left[w ; w_{-}, w_{+}\right]$be the linear function interpolating $\left(f(w) \bullet a_{x}\right)$ at $w=w_{-}$and $w=w_{+}$. It is easy to see that the condition (2.4) for all $c$ is equivalent to the following inequality:

$$
\operatorname{sign}\left(w_{+}-w_{-}\right)\left(f\left[w ; w_{-}, w_{+}\right]-\left(f(w) \bullet a_{x}\right)\right) \leq 0
$$

for all $w$ between $w_{-}$and $w_{+}$. In the case of one spatial dimension, (2.2), (2.1) and (2.5) are reduced to

$$
\begin{aligned}
& W(x, t)= \begin{cases}w_{-} & \text {if } x<s t, \\
w_{+} & \text {if } x>s t,\end{cases} \\
& s\left(w_{+}-w_{-}\right)=f\left(w_{+}\right)-f\left(w_{-}\right),
\end{aligned}
$$

and

$$
\operatorname{sign}\left(w_{+}-w_{-}\right)\left(f\left[w ; w_{-}, w_{+}\right]-f(w)\right) \leq 0
$$

respectively. In the last inequality, we have scaled $a$ so that $a_{x}=1$.

To see the properties of discontinuities of BV weak solutions of conservation laws, let us review some aspects of the BV solution theory of Vol'pert [26]. For any set $E \subset \mathbf{R}^{n}, \mu(E)$ is its Lebesgue measure. With $B_{r}\left(x_{0}\right)$ we denote the ball centered at $x_{0}$ with the radius $r$. Let $a$ be a unit vector in $\mathbf{R}^{n}$, and $R_{a}\left(x_{0}\right)$ be the half space $\left(\left(x-x_{0}\right) \bullet a\right)>0$ in $\mathbf{R}^{n}$. A point of density (resp. rarefaction) for the set $E$ is a point $x$ for which

$$
\lim _{r \rightarrow 0} \mu\left(E \cap B_{r}(x)\right) / \mu\left(B_{r}(x)\right)=1 \text { (resp. 0). }
$$

If $w(x)$ is a function defined on a set $E \subset \mathbf{R}^{n}$ and $x_{0}$ is not a point of rarefaction for $E$, then $L_{E} w\left(x_{0}\right)$ will denote the approximate limit of the function $w(x)$ at the point $x_{0}$ with respect to the set $E: \forall \varepsilon>0, x_{0}$ is a point of rarefaction of the set

$$
\left\{x:\left|w(x)-L_{E} w\left(x_{0}\right)\right|>\varepsilon, x \in E\right\} .
$$


Definition 2.1. Let $w(x)$ be a function defined on $\mathbf{R}^{n}$.

$(\alpha)$ A point $x_{0} \in \mathbf{R}^{n}$ is said to be regular if there exists a unit vector $a$ such that $l_{a} w\left(x_{0}\right)$ and $l_{-a} w\left(x_{0}\right)$ exist and are finite. Here, $l_{a} w\left(x_{0}\right)=L_{R_{a}\left(x_{0}\right)} w\left(x_{0}\right)$.

$(\beta)$ The point $x_{0}$ is said to be a point of jump for $w(x)$ if it is regular and $l_{a} w\left(x_{0}\right) \neq l_{-a} w\left(x_{0}\right)$. The set of the jump points for $w(x)$ is denoted by $\Gamma(w)$.

$(\gamma)$ If $x_{0} \in \Gamma(w)$, then the value $a$ appearing in the definition $(\alpha)$ is called the normal to $\Gamma(w)$ at the point $x_{0}$. We let $a=\left(a_{t}, a_{x}\right)$ where $a_{t}$ is the time component of $a$, and $a_{x}$ the space component.

We now apply the preceding concepts for the BV functions of $n+1$ variables to a BV weak solution $w(x, t)$ of the conservation law. For any $\left(x_{0}, t_{0}\right) \in \Gamma(w)$, let $a$ be the normal to $\Gamma(w)$ at the point $\left(x_{0}, t_{0}\right)$. Let $w_{+}=l_{a} w\left(x_{0}, t_{0}\right), w_{-}=l_{-a} w\left(x_{0}, t_{0}\right)$. We then call $W$, defined by (2.2), the traveling discontinuity associated with $w$ at the jump point $\left(x_{0}, t_{0}\right)$.

Denote by $H_{n}$ the $n$-dimensional Hausdorff measure. The following basic result holds:

Lemma 2.2 ( $\mathrm{Vol}^{\prime}$ pert [26]). A necessary and sufficient condition for a weak solution $w \in B V$ of $w_{t}+f(w)_{x}=0$ to be an entropy solution is that (2.4) holds for $H_{n}$-almost all points in $\Gamma(w)$.

Throughout this paper similarity transforms and the self-similar property of the schemes play important roles. Let $\mathcal{S}_{x_{0}, t_{0}}^{\varepsilon}$ be the similarity transform centered at a point $\left(x_{0}, t_{0}\right)$ :

$$
\mathcal{S}_{x_{0}, t_{0}}^{\varepsilon}((x, t))=\left(x_{0}+\varepsilon x, t_{0}+\varepsilon t\right) .
$$

This induces a transform $T_{x_{0}, t_{0}}^{\varepsilon}$ in the set of the functions $\psi$ defined on a domain $\Omega \subset \mathbf{R}^{n} \times \mathbf{R}$ :

$$
T_{x_{0}, t_{0}}^{\varepsilon} \psi=\left.\psi \circ \mathcal{S}_{x_{0}, t_{0}}^{\varepsilon}\right|_{\Omega}
$$

if $\mathcal{S}_{x_{0}, t_{0}}^{\varepsilon} \Omega \subset \Omega$, where $\left.\phi\right|_{\Omega}$ denotes the restriction of $\phi$ to the set $\Omega$.

Define $w_{\varepsilon}(x, t)$ by

$$
\begin{aligned}
w_{\varepsilon}(x, t) & =\left(T_{x_{0}, t_{0}}^{\varepsilon} w\right)(x, t) \\
& =w \circ \mathcal{S}_{x_{0}, t_{0}}^{\varepsilon}((x, t)) \\
& =w\left(x_{0}+\varepsilon x, t_{0}+\varepsilon t\right)
\end{aligned}
$$

We make the following preliminary observation, which turns out to be one of the foundations of the WEI method.

Lemma 2.3. Let $\left(x_{0}, t_{0}\right)$ be a jump point of a $B V$ weak solution $w$ in the sense of Definition 2.1. If $\left\{\varepsilon_{k}\right\}_{k=1}^{\infty}$ is a sequence of positive numbers such that $\lim _{k \rightarrow \infty} \varepsilon_{k}=$ 0 , then the sequence $\left\{w_{\varepsilon_{k}}\right\}$ converges in $L_{\text {loc }}^{1}$ to the traveling discontinuity $W$ associated with the jump point $\left(x_{0}, t_{0}\right)$.

Proof. Fix any positive number $R$; by the definition of the jump points, for any $\delta>0$, there exists a number $\varepsilon(\delta)$ such that if $r<\varepsilon(\delta) R$,

$$
\frac{\mu\left(\left\{(x, t):\left|w(x, t)-W\left(\frac{x-x_{0}}{\varepsilon(\delta)}, \frac{t-t_{0}}{\varepsilon(\delta)}\right)\right|>\frac{\delta}{2 V_{R}}\right\} \cap B_{r}\left(x_{0}, t_{0}\right)\right)}{\mu\left(B_{r}\left(x_{0}, t_{0}\right)\right)}<\frac{\delta}{4 M V_{R}},
$$


where $V_{\gamma}=\mu\left(B_{\gamma}(0,0)\right)$ for any $\gamma>0$. Therefore, for all $k$ with $\varepsilon_{k}<\varepsilon(\delta)$ and $r_{k}=\varepsilon_{k} R$,

$$
\begin{aligned}
\int_{B_{R}} & \left|w_{\varepsilon_{k}}(x, t)-W(x, t)\right| d x d t \\
& =\left(\int_{B_{r_{k}}\left(x_{0}, t_{0}\right)}\left|w(x, t)-W\left(\frac{x-x_{0}}{\varepsilon_{k}}, \frac{t-t_{0}}{\varepsilon_{k}}\right)\right| d x d t\right) \frac{\mu\left(B_{R}(0,0)\right)}{\mu\left(B_{r_{k}}\left(x_{0}, t_{0}\right)\right)} \\
& <2 M V_{r_{k}} \frac{V_{R}}{V_{r_{k}}} \frac{\delta}{4 M V_{R}}+\frac{\delta}{2 V_{R}} V_{r_{k}} \frac{V_{R}}{V_{r_{k}}} \\
& =\delta .
\end{aligned}
$$

This proves the lemma.

\section{WEI CRITERIA FOR CONVERGENCE}

In this section, we present, in order of ascending practicability but descending generality, four WEI criteria for convergence of semidiscrete TVB and TVD schemes. The first one is based on Lemma 2.3, and each successive one is based on the preceding one. The relatively easy proofs of the first two and the last are contained in this section. The proof of the third is contained in the Supplement at the end of this issue.

The first criterion, valid for conservation laws $w_{t}+f(w)_{x}=0$ in several spatial dimensions, is as follows.

Theorem 3.1. (A necessary and sufficient condition for convergence of TVB schemes) A TVB scheme (1.2)-(1.4) for the Cauchy problem (1.1) converges if and only if there exists no sequence of numerical solutions of the scheme that converge in $L_{\mathrm{loc}}^{1}\left(\mathbf{R} \times \mathbf{R}^{+}\right)$to a traveling expansion shock.

Proof. The "only if" part is trivial. For the "if" part, we argue by contradiction. Assume that a TVB self-similar scheme (1.2)-(1.4) does not converge for some given BV initial condition. The Lax-Wendroff Theorem implies that there is a subsequence $\left\{u^{\nu}\right\}_{\nu=1}^{\infty}$ that converges in $L_{\text {loc }}^{1}\left(\mathbf{R}^{n} \times \mathbf{R}^{+}\right)$to a weak solution $w$ of (1.1) that is not the entropy solution. Suppose that the space step of $u^{\nu}$ is $h_{\nu}$, and that $\lim _{\nu \rightarrow \infty} h_{\nu}=0$. Lemma 2.2 implies that there is a jump point $\left(x_{0}, t_{0}\right)$ of $w$ that violates the entropy condition (2.4). Let $W$ be the expansion shock associated with $w$ at $\left(x_{0}, t_{0}\right)$. Let $\left\{R_{k}\right\}_{k=1}^{\infty}$ be an increasing sequence of positive numbers such that $\lim _{n \rightarrow \infty} R_{n}=\infty$. First, by Lemma 2.3 , for any $k$, one can choose a sufficiently small positive number $\varepsilon_{k}$ so that

$$
\int_{B_{R_{k}}}\left|w_{\varepsilon_{k}}(x, t)-W(x, t)\right| d x d t<\frac{1}{2^{k+1}} .
$$

Next, since the scheme is self-similar, for all $\varepsilon>0,\left\{T_{x_{0}, t_{0}}^{\varepsilon} u^{k}\right\}_{k=1}^{\infty}$ is also a sequence of numerical solutions of the same scheme, and it converges to $w_{\varepsilon}$ in $L_{\mathrm{loc}}^{1}\left(\mathbf{R}^{n} \times \mathbf{R}^{+}\right)$ as $k \rightarrow \infty$. Hence, one can choose $\nu_{k}$ so that both

$$
\int_{B_{R_{k}}}\left|T_{x_{0}, t_{0}}^{\varepsilon_{k}} u^{\nu_{k}}(x, t)-w_{\varepsilon_{k}}(x, t)\right|<\frac{1}{2^{k+1}}
$$


and $\frac{h_{\nu_{k}}}{\varepsilon_{k}}<\frac{1}{2^{k}}$ hold for each $k$. These two inequalities imply that

$$
\int_{B_{R_{k}}}\left|T_{x_{0}, t_{0}}^{\varepsilon_{k}} u^{\nu_{k}}(x, t)-W(x, t)\right|<\frac{1}{2^{k}} .
$$

Now clearly, $\left\{T_{x_{0}, t_{0}}^{\varepsilon_{k}} u^{\nu_{k}}\right\}_{k=1}^{\infty}$ converges to $W$ in $L_{\text {loc }}^{1}\left(\mathbf{R}^{n} \times \mathbf{R}^{+}\right)$.

For TVD self-similar schemes of one spatial dimension, we may enhance our result by using the property that the total spatial variation of $u\left(x_{0}+\varepsilon x, t_{0}+\varepsilon\right)$ is the same as that of $u\left(x, t_{0}+\varepsilon\right)$ for any function $u$ with bounded total spatial variation. In the following, we suppose $T V_{u}(t)$ is the total spatial variation of $u$ at the time $t, D T V_{u}\left(t_{1}, t_{2}\right) \stackrel{\text { def }}{=} T V_{u}\left(t_{1}\right)-T V_{u}\left(t_{2}\right)$ is the decay of $T V_{u}(t)$ from $t_{1}$ to $t_{2},\left\{\varepsilon_{k}\right\}_{k=1}^{\infty}$ is a sequence of positive numbers such that $\lim _{k \rightarrow \infty} \varepsilon_{k}=0$, w- and $w_{+}$are two distinct real constants in the domain of $f$, and $W(x, t)$ is a traveling discontinuity defined by (2.2) with the two states $w_{-}$and $w_{+}$. For any constant $B>0$, we define $\widehat{\Psi}_{w_{-}, w_{+}, B}$ to be the set of the sequences of numerical solutions $\left\{u^{k}\right\}_{k=1}^{\infty}$ generated by a TVD self-similar scheme, such that the following conditions hold:

(i) $u^{k}(x, t) \rightarrow W(x, t)$ in $L_{\text {loc }}^{1}\left(\mathbf{R} \times \mathbf{R}^{+}\right)$as $k \rightarrow \infty$,

(ii) $\left|T V_{u^{k}}(t)\right|<B$ for all $t$ and $k$, and

(iii) $D T V_{u^{k}}(0,1)<\varepsilon_{k}$ for each $k$.

We call $W(x, t)$ the limit of $\widehat{\Psi}_{w_{-}, w_{+}, B}$, and $w_{-}, w_{+}$the two states of $\widehat{\Psi}_{w_{-}, w_{+}, B}$. Here is our second criterion for convergence.

Theorem 3.2. (A necessary and sufficient condition for convergence of TVD schemes) A TVD self-similar scheme given by (1.2)-(1.4) for conservation laws of one spatial variable converges if and only if for all triples of numbers $\left\{w_{-}, w_{+}, B\right\}$ with $B>0$, the set $\widehat{\Psi}_{w_{-}, w_{+}, B}=\emptyset$ when its limit $W(x, t)$ is a traveling expansion shock.

Proof. Again, the "only if" part is trivial. It suffices to prove the contrapositive of the "if" part. Assume the convergence of a TVD self-similar scheme given by (1.2)(1.4) fails. It follows from Theorem 3.1 that there exists a sequence of numerical solutions $\left\{u^{\nu}\right\}$ of the scheme that converges in $L_{\text {loc }}^{1}\left(\mathbf{R} \times \mathbf{R}^{+}\right)$to a traveling expansion shock $W$ of the form (2.6). Moreover, the total spatial variations of the numerical solutions are bounded by a positive number $B$. Since the scheme is TVD, for any positive integers $n$ and $\nu$, there is an integer $m(n, \nu)$ such that $0 \leq m(n, \nu) \leq n-1$ and

$$
D T V_{u^{\nu}}(m(n, \nu) / n,(m(n, \nu)+1) / n) \leq \frac{1}{n} D T V_{u^{\nu}}(0,1) \leq B / n .
$$

Let $t_{n, \nu}=m(n, \nu) / n$, and $x_{n, \nu}=s t_{n, \nu}$. For each $k$, one can first choose a sufficiently large $n=n_{k}$ so that $B / n_{k}<\varepsilon_{k}$. Then, since $u^{\nu} \rightarrow W$ in $L_{\text {loc }}^{1}\left(\mathbf{R} \times \mathbf{R}^{+}\right)$, one can choose a sufficiently large $\nu=\nu_{k}$ so that

$$
\int_{0}^{1} \int_{s t-1}^{s t+1}\left|u^{\nu_{k}}(x, t)-W(x, t)\right| d x d t<\varepsilon_{k} / n_{k}^{2} .
$$

For simplicity we set $\hat{x}_{k}=x_{n_{k}, \nu_{k}}, \hat{t}_{k}=t_{n_{k}, \nu_{k}}$, and $\hat{u}^{k}(x, t)=T_{\hat{x}_{k}, \hat{t}_{k}}^{1 / n_{k}} u^{\nu_{k}}(x, t)$. We then have

$$
\int_{0}^{n_{k}} \int_{s t-n_{k}}^{s t+n_{k}}\left|\hat{u}^{k}(x, t)-W(x, t)\right| d x d t<\varepsilon_{k}
$$


since $T_{s \alpha, \alpha}^{c} W(x, t)=W(x, t)$ for any positive constants $\alpha$ and $c$. Moreover, $T V_{\hat{u}^{k}}(t)$ $\leq B$ and $D T V_{\hat{u}^{k}}(0,1)=D T V_{u^{\nu}}(m(n, \nu) / n,(m(n, \nu)+1) / n)<\varepsilon_{k}$ hold since the total spatial variation of $u\left(x_{0}+\varepsilon x, t_{0}+\varepsilon\right)$ is the same as that of $u\left(x, t_{0}+\varepsilon\right)$ for any function $u$ with bounded total spatial variation. Therefore, $\left\{\hat{u}^{k}\right\}_{k=1}^{\infty} \in \widehat{\Psi}_{w_{-}, w_{+}, B}$. The lemma is proved.

Remark. It is not clear whether the same result holds for $n$ spatial dimensions because $T_{x_{0}, t_{0}}^{\varepsilon}$ increases the spatial variation by a factor $\varepsilon^{1-n}$.

Our next two criteria demand the following TVD condition of Tadmor [25].

Assumption 3.3. The numerical fluxes $g_{j+\frac{1}{2}}(t), j=0, \pm 1, \pm 2, \ldots$, satisfy

$$
g_{j+\frac{1}{2}}(t) \geq f\left(u_{j}\right) \geq g_{j-\frac{1}{2}}(t) \quad \text { if } u_{j}(t)-u_{j \pm 1}(t) \geq 0,
$$

and

$$
g_{j+\frac{1}{2}}(t) \leq f\left(u_{j}\right) \leq g_{j-\frac{1}{2}}(t) \quad \text { if } u_{j}(t)-u_{j \pm 1}(t) \leq 0 .
$$

Corollary 3.4. Let $u$ be a scheme of the form (1.2)-(1.4) that satisfies Assumption 3.3 , and let $\varepsilon$ be a positive constant.

(i) If

$$
\min \left(\operatorname{sign}\left(\Delta_{+} u_{j}(t)\right) \Delta_{-} u_{j}(t),\left|\Delta_{+} u_{j}(t)\right|\right)<\varepsilon
$$

holds for an integer $j$, then

$$
\left|g_{j \pm \frac{1}{2}}(t)-f\left(u_{j}(t)\right)\right|<\left|g_{j+\frac{1}{2}}(t)-g_{j-\frac{1}{2}}(t)\right|+C \varepsilon .
$$

(ii) If

$$
\min \left(\left|\Delta_{-} u_{j}(t)\right|,\left|\Delta_{+} u_{j}(t)\right|\right)<\varepsilon
$$

holds for an integer $j$, then

$$
\left|g_{j \pm \frac{1}{2}}(t)-f\left(u_{j}(t)\right)\right|<C \varepsilon .
$$

Proof. First suppose (3.1) holds for some $j$. It is easy to see that by suitably adding $\varepsilon$ to or subtracting it from $u_{j+1}(t)$ or $u_{j-1}(t)$ when necessary, and denoting the modified $u$ by $\bar{u}$ and the correspondingly modified $g_{j \pm \frac{1}{2}}(t)$ by $\bar{g}_{j \pm \frac{1}{2}}(t)$, one can achieve $\Delta_{-} \bar{u}_{j}(t) \Delta_{+} \bar{u}_{j}(t)<0$. Then, by Assumption 3.3 ,

$$
\left|\bar{g}_{j \pm \frac{1}{2}}(t)-f\left(u_{j}(t)\right)\right| \leq\left|\bar{g}_{j+\frac{1}{2}}(t)-\bar{g}_{j-\frac{1}{2}}(t)\right| .
$$

Since the numerical flux function $g$ is Lipschitz continuous, $\left|\bar{g}_{j \pm \frac{1}{2}}(t)-g_{j \pm \frac{1}{2}}(t)\right|<C \varepsilon$. Therefore, the conclusion of (i) follows from (3.3) and the triangle inequality.

Next, one can prove (ii) similarly by using the fact that, because of $(3.2), u_{j}(t)$ becomes a local spatial minimum ( maximum ) if one adds ( subtracts) $\varepsilon$ to (from) both $u_{j-1}(t)$ and $u_{j+1}(t)$.

We need to introduce the concepts of asymptotic traveling discontinuities and asymptotic traveling expansion shocks ( we use "asymptotic" instead of "discrete" to avoid a confusion with Jennings's discrete shocks [11]). For this purpose, we need the following notion of paths to be the boundaries of the transition areas of the discontinuities.

Definition 3.5. A gridpoint-valued function $x_{I(t)}=I(t) h+c, 0 \leq t \leq 1$, is said to be an $\varepsilon$-path of the first type with respect to $u$ if the following conditions hold: 
(i) There is a finite partition of $[0,1]$ :

$$
0=\tau_{0}<\tau_{1}<\cdots<\tau_{n}=1
$$

such that $I(t)$ is a constant integer on each subinterval $\left(\tau_{\nu-1}, \tau_{\nu}\right)$.

(ii) For all $j$ between $I\left(\tau_{\nu}-\right)$ and $I\left(\tau_{\nu}+\right), u_{j}\left(\tau_{\nu}\right)=u_{I\left(\tau_{\nu}-\right)}\left(\tau_{\nu}\right)=u_{I\left(\tau_{\nu}+\right)}\left(\tau_{\nu}\right)$. Moreover, $u_{I\left(\tau_{\nu}+\right)}\left(\tau_{\nu}\right)$ is monotone with respect to $\nu$.

(iii) The inequality $\min \left(\operatorname{sign}\left(\Delta_{+} u_{I(t)}(t)\right) \Delta_{-} u_{I(t)}(t),\left|\Delta_{+} u_{I(t)}(t)\right|\right)<\varepsilon$ holds.

(iv) The total variation of the function $u_{I(t)}(t)$ of $t$ for $0 \leq t \leq 1$ is bounded by $\varepsilon$.

Definition 3.6. A gridpoint-valued function $x_{I(t)}=I(t) h+c$ is said to be an $\varepsilon$-path of the second type with respect to $u$ if the following conditions hold:

(i) $I(t)$ is a monotone function of $\mathrm{t}$ on the interval $[0,1]$ with the property that there is a finite partition of $[0,1]$ :

$$
0=\tau_{0}<\tau_{1}<\cdots<\tau_{n}=1
$$

such that $I(t)$ is a constant integer on each subinterval $\left(\tau_{\nu-1}, \tau_{\nu}\right)$. Moreover, $\left|I\left(\tau_{\nu}+\right)-I\left(\tau_{\nu}-\right)\right|=1$ for $\nu=1, \cdots, n-1$.

(ii) There is a constant $A$ such that for any $t \in[0,1],\left|u_{j}(t)-A\right|<\varepsilon$ holds if $x_{j}$ is in the stencil of the scheme at $\left(x_{I(t)}, t\right)$.

We have the following lemma relating the numerical flux to the exact flux along an $\varepsilon$-path of either type.

Lemma 3.7. Suppose that $\left\{u_{j}(t)\right\}_{j=-\infty}^{\infty}$ is a numerical solution generated by a scheme (1.2)-(1.4) that satisfies Assumption 3.3. Let $x_{I(t)}=I(t) h+c$ to be an $\varepsilon$-path of either type. We then have

$$
\int_{0}^{1}\left|g_{I(t) \pm \frac{1}{2}}(t)-f\left(u_{I}(t)\right)\right| d t<C \varepsilon
$$

where $C$ depends on the Lipschitz coefficients of $g$ only.

Proof. The conclusion for an $\varepsilon$-path of the second type follows directly from the consistency relation (1.4), the Lipschitz condition of the numerical flux, and condition (ii) in Definition 3.6. For an $\varepsilon$-path of the first type, using (1.2) and conditions (i), (ii) and (iv) in Definition 3.5, we get

$$
\int_{0}^{1}\left|g_{I(t)+\frac{1}{2}}(t)-g_{I(t)-\frac{1}{2}}(t)\right| d t=h \int_{0}^{1}\left|\frac{d}{d t} u_{I(t)}(t)\right| d t \leq \varepsilon h .
$$

With condition (iii) in Definition 3.5, apply Corollary 3.4 and obtain

$$
\left|g_{I(t) \pm \frac{1}{2}}(t)-f\left(u_{I(t)}(t)\right)\right|<\left|g_{I(t)+\frac{1}{2}}(t)-g_{I(t)-\frac{1}{2}}(t)\right|+C \varepsilon
$$

We complete the proof by integrating the last inequality from 0 to 1 and applying (3.5).

Let $\left\{u_{j}^{k}(t), j=0, \pm 1, \pm 2, \ldots\right\}_{k=1}^{\infty}$ be a sequence of numerical solutions generated by a semidiscrete schemes associated with a sequence of meshes $\left\{x_{j}^{k}, j=\right.$ $0, \pm 1, \pm 2, \cdots\}_{k=1}^{\infty}$, where $x_{j}^{k}=j h_{k}+c_{k}$ and $h_{k} \rightarrow 0$ as $k \rightarrow \infty$. Let $\left\{\varepsilon_{k}\right\}$ be a sequence of positive numbers such that $\varepsilon_{k} \rightarrow 0$ as $k \rightarrow \infty$.

Definition 3.8. A sequence of pairs of $\varepsilon_{k}$-paths of either type, $\left\{x^{(k)}(t), y^{(k)}(t)\right\}_{k=1}^{\infty}$, where $x^{(k)}(t)=x_{I^{k}(t)}^{k}=I^{k}(t) h_{k}+c_{k}, y^{(k)}(t)=x_{J^{k}(t)}^{k}=J^{k}(t) h_{k}+c_{k}$, and $0 \leq t \leq 1$, is said to be an asymptotic traveling wave $(A T W)$ of $\left\{u^{k}\right\}$ if $x^{(k)}(t)<y^{(k)}(t)$, and 
if there is a linear function $x(t)=s t+r$ and two distinct constants $L$ and $R$ such that:

(i) In the case $s=0$, for each $k$, if either path of the pair $\left\{x^{(k)}(t), y^{(k)}(t)\right\}$, say $x^{(k)}(t)$, is of the second type, then $x^{(k)}(t)$ is a constant depending only on $k$.

(ii) Both $x^{(k)}(t)$ and $y^{(k)}(t)$ converge to $x(t)$ uniformly on the $t$-interval $[0,1]$ as $k \rightarrow \infty$.

(iii) $u_{I^{k}(t)}^{k}(t)$ and $u_{J^{k}(t)}^{k}(t)$ converge to $L$ and $R$, respectively, on the $t$-interval $[0,1]$ as $k \rightarrow \infty$.

For each $k$, denote by $\widehat{\Omega}^{k}$ the region $x_{I^{k}(t)}-h_{k} / 2<x<x_{J^{k}(t)}-h_{k} / 2,0 \leq t \leq 1$. We call the sequence $\left\{\widehat{\Omega}^{k}\right\}$ the transition region of the $A T W, x(t)$ the limit path of the $A T W, L$ and $R$ the two states of the $A T W$, and $|R-L|$ the amplitude of the $A T W$.

Next we consider entropy properties of an ATW. Following Osher [17], for any convex entropy $U(w)$ and its flux $F(w)$, we adopt the numerical entropy flux

$$
G_{j-\frac{1}{2}}(t) \stackrel{\text { def }}{=} F\left(u_{j}\right)+U^{\prime}\left(u_{j}\right)\left[g_{j-\frac{1}{2}}-f\left(u_{j}\right)\right] .
$$

By Lemma 3.7, $G_{j-\frac{1}{2}}(t)$ satisfies the following

Corollary 3.9. If the numerical scheme satisfies the conditions of Lemma 3.7, then

$$
\int_{0}^{1}\left|G_{I(t)-\frac{1}{2}}(t)-F\left(u_{I(t)}(t)\right)\right| d t<C \varepsilon
$$

where $x_{I(t)}=I(t) h+c$ is an $\varepsilon$-path of either type.

In [17], Osher proved the following equality:

$$
h\left(\frac{d}{d t} U\left(u_{j}(t)\right)+D_{+} G_{j-\frac{1}{2}}(t)\right)=\int_{u_{j}(t)}^{u_{j+1}(t)} U^{\prime \prime}(w)\left(g_{j+\frac{1}{2}}-f(w)\right) d w .
$$

Let $\phi(x, t)$ be a smooth function with a compact support in the domain $-\infty<$ $x<\infty, 0<t<1$. Set $\phi_{j}(t)=\phi\left(x_{j}, t\right)$. Consider

$$
\widehat{\Phi}^{k} \stackrel{\text { def }}{=} \int_{0}^{1} \sum_{j=I^{k}(t)}^{J^{k}(t)-1} h_{k}\left(\frac{d}{d t} U\left(u_{j}^{k}(t)\right)+D_{+} G_{j-\frac{1}{2}}^{k}(t)\right) \phi_{j}(t) d t,
$$

We have the following fundamental result.

Lemma 3.10. Suppose that $\left\{u_{j}(t)\right\}_{j=-\infty}^{\infty}$ is a numerical solution generated by a scheme (1.2)-(1.4) that satisfies Assumption 3.3. Let $\left\{I^{k}(t) h_{k}+c_{k}, J^{k}(t) h_{k}+c_{k}\right\}$ be an $A T W$ of $\left\{u^{k}\right\}$ with the limit path $x(t)=s t+r$ and the two states $L$ and $R$. We have

$$
\lim _{k \rightarrow \infty} \widehat{\Phi}^{k}=[F(R)-F(L)-s(U(R)-U(L))] \int_{x=s t} \phi(x, t) d t,
$$

where $\widehat{\Phi}^{k}$ is defined by (3.8).

Proof. Without loss of generality, assume that $r=0$. Let

$$
\widehat{\Phi}_{1}^{k}=\int_{0}^{1} \sum_{j=I^{k}(t)}^{J^{k}(t)-1} h_{k} \frac{d}{d t} U\left(u_{j}^{k}(t)\right) \phi_{j}(t) d t
$$


and

$$
\widehat{\Phi}_{2}^{k}=\int_{0}^{1} \sum_{j=I^{k}(t)}^{J^{k}(t)-1} h_{k} D_{+} G_{j-\frac{1}{2}}^{k}(t) \phi_{j}(t) d t .
$$

It suffices to prove

$$
\widehat{\Phi}_{1}^{k} \rightarrow-s(U(R)-U(L)) \int_{x=s t} \phi(x, t) d t
$$

and

$$
\widehat{\Phi}_{2}^{k} \rightarrow(F(R)-F(L)) \int_{x=s t} \phi(x, t) d t .
$$

A summation by parts of $\widehat{\Phi}_{2}^{k}$ leads to

$$
\begin{aligned}
\widehat{\Phi}_{2}^{k}= & -\int_{0}^{1}\left(\sum_{j=I^{k}(t)+1}^{J^{k}(t)-1} G_{j-\frac{1}{2}}^{k}(t) \Delta_{-} \phi_{j}(t)\right) d t \\
& +\int_{0}^{1}\left(G_{J^{k}(t)-\frac{1}{2}}^{k}(t) \phi_{J^{k}(t)-1}(t)-G_{I^{k}(t)-\frac{1}{2}}^{k}(t) \phi_{I^{k}(t)}(t)\right) d t
\end{aligned}
$$

The first integral in (3.12) tends to 0 since $G_{j-\frac{1}{2}}^{k}(t)$ is uniformly bounded, $\left|\Delta_{-} \phi_{j}\right| \leq$ $C h_{k}$, and $\lim _{k \rightarrow \infty}\left(J^{k}(t)-I^{k}(t)\right) h_{k}=0$. The second integral tends to

$$
\int_{x=s t}(F(R)-F(L)) \phi(x, t) d t
$$

since Corollary 3.9 implies that $G_{J^{k}(t)-\frac{1}{2}}^{k}(t) \rightarrow F(R)$ and $G_{I^{k}(t)-\frac{1}{2}}^{k}(t) \rightarrow F(L)$ respectively in $L^{1}([0,1])$, and since condition (ii) in Definition 3.8 implies that both $\phi_{J^{k}(t)-1}(t)$ and $\phi_{I^{k}(t)}(t)$ tend to $\phi(s t, t)$ uniformly for $0 \leq t \leq 1$. This completes the proof of (3.11).

We now turn to the proof of (3.10). Without loss of generality, let $x_{J^{k}(t)}^{k}=$ $J^{k}(t) h_{k}+c_{k}$ be $\varepsilon_{k}$-paths of the first type, and let $x_{I^{k}(t)}^{k}=I^{k}(t) h_{k}+c_{k}$ be $\varepsilon_{k}$-paths of the second type.

Consider separately the following three situations, which exhaust all possibilities:

(i) $s>0$. We notice that $\phi(x, t)$ has compact support $\operatorname{spt}(\phi)$ in $\left\{(x, t) \in \mathbf{R} \times \mathbf{R}^{+}\right.$: $0 \leq t \leq 1\}$ : Define

and

$$
\begin{aligned}
I_{\max }^{k} & =\max _{t}\left\{I^{k}(t):\left(x_{I^{k}(t)}^{k}, t\right) \in \operatorname{spt}(\phi)\right\}, \\
J_{\max }^{k} & =\max _{t}\left\{J^{k}(t):\left(x_{J^{k}(t)}^{k}, t\right) \in \operatorname{spt}(\phi)\right\}, \\
I_{\min }^{k} & =\min _{t}\left\{I^{k}(t):\left(x_{I^{k}(t)}^{k}, t\right) \in \operatorname{spt}(\phi)\right\},
\end{aligned}
$$

$$
J_{\min }^{k}=\min _{t}\left\{J^{k}(t):\left(x_{J^{k}(t)}^{k}, t\right) \in \operatorname{spt}(\phi)\right\} .
$$

It follows that for sufficiently large $k$, and for the $x_{j}^{k}$ such that

$$
\min \left(I_{\min }^{k}, J_{\min }^{k}\right) \leq j \leq \max \left(I_{\max }^{k}, J_{\max }^{k}\right),
$$

the line segment $\left\{\left(x_{j}^{k}, t\right): 0 \leq t \leq 1\right\}$ crosses each boundary of $\widehat{\Omega}^{k}$ an odd number of times. Suppose that it crosses the right boundary at

$$
t=\underline{t}_{1}^{k, j}, \ldots, \underline{t}_{2 \theta_{j}^{k}+1}^{k, j}
$$


and that $\underline{u}_{i}^{k, j} \stackrel{\text { def }}{=} u_{j}^{k}\left(\underline{t}_{i}^{k, j}\right)$. Suppose also that it crosses the left boundary at $\bar{t}^{k, j}$, and that $\bar{u}^{k, j} \stackrel{\text { def }}{=} u_{j}^{k}\left(\bar{t}^{k, j}\right)$. An integration by parts leads to

$$
\begin{aligned}
\widehat{\Phi}_{1}^{k}= & -\int_{0}^{1} \sum_{j=I^{k}(t)}^{J^{k}(t)-1} h_{k} U\left(u_{j}^{k}(t)\right) \frac{d}{d t} \phi_{j}(t) d t+\sum_{j=\min _{t}\left(I^{k}(t)\right)}^{\max _{t}\left(J^{k}(t)-1\right)} h_{k} U\left(\bar{u}^{k, j}\right) \phi_{j}\left(\bar{t}^{k, j}\right) \\
& -\sum_{j=\min _{t}\left(I^{k}(t)\right)}^{\max _{t}\left(J^{k}(t)-1\right)} h_{k} \sum_{i=1}^{2 \theta_{j}^{k}+1}(-1)^{i+1} U\left(\underline{u}_{i}^{k, j}\right) \phi_{j}\left(\underline{t}_{i}^{k, j}\right) .
\end{aligned}
$$

The first term on the right tends to zero since $\left(x_{J^{k}(t)}^{k}-x_{J^{k}(t)}^{k}\right) \rightarrow 0$ uniformly, and $U\left(u_{j}^{k}(t)\right) \frac{d}{d t} \phi_{j}(t)$ is uniformly bounded. The second term tends to $U(L) \int_{x=s t} \phi d x$ since $\bar{u}^{k, j} \rightarrow L$ uniformly. The third term tends to $-U(R) \int_{x=s t} \phi d x$ since, by condition (iv) of Definition 3.5, the total variation of $U\left(\underline{u}_{i}^{k, j}\right)$ in $i$ tends to zero uniformly in $j$, and since $U\left(\underline{u}_{i}^{k, j}\right)$ tends to $U(R)$ uniformly in $i$ and $j$. This proves (3.10) in the case $s>0$.

(ii) $s<0$. The proof is parallel to that of case (i) and is omitted.

(iii) $s=0$. We assume that the line segment $\left\{\left(x_{j}^{k}, t\right): t^{\prime} \leq t \leq t^{\prime \prime}\right\}$ crosses the right boundary at

$$
t=\underline{t}_{1}^{k, j}, \ldots, \underline{t}_{\pi_{j}^{k}}^{k, j} .
$$

Proceed similarly to case (i). Since $I^{k}(t)$ is a constant ( see (i) in Definition 3.8), it suffices to consider the term

$$
-\sum_{j=\min _{t}\left(I^{k}(t)\right)}^{\max _{t}\left(J^{k}(t)-1\right)} h_{k} \sum_{i=1}^{\pi_{j}^{k}}(-1)^{i+1} \omega_{j} U\left(\underline{u}_{i}^{k, j}\right) \phi_{j}\left(\underline{t}_{i}^{k, j}\right),
$$

where $\omega_{j}$ for each $j$ is a real constant with $\left|\omega_{j}\right|=1$. Since the total variation of $U\left(\underline{u}_{i}^{k, j}\right)$ with respect to $i$ is uniformly bounded,

$$
\left|\sum_{i=1}^{\pi_{j}^{k}}(-1)^{i+1} \omega_{j} U\left(\underline{u}_{i}^{k, j}\right) \phi_{j}\left(\underline{t}_{i}^{k, j}\right)\right|
$$

has an upper bound that is independent of $k$ and $j$. Therefore, the term (3.14) tends to zero since in this case $h_{k}\left(\max _{t}\left(J^{k}(t)-\min _{t}\left(I^{k}(t)\right)\right)\right.$ tends to zero. This proves (3.10) in the case $s=0$, and completes our proof of the lemma.

Corollary 3.11. With the conditions of Lemma 3.10, we have the following discrete Rankine-Hugoniot condition:

$$
f(R)-f(L)=s(R-L) .
$$

Moreover,

$$
\lim _{k \rightarrow \infty} \widehat{\Phi}^{k}=\int_{L}^{R} U^{\prime \prime}(w)(f[w ; L, R]-f(w)) d w \int_{x=s t} \phi(x, t) d t .
$$

Proof. First, choose $U(w)=w$, and $F(w)=f(w)$. We have

$$
G_{j+\frac{1}{2}}^{k}(t)=g_{j+\frac{1}{2}}^{k}(t) .
$$

This and the definition of the numerical scheme imply that $\widehat{\Phi}^{k} \equiv 0$. Hence, the relation (3.9) becomes the discrete Rankine-Hugoniot condition (3.15). 
Next, for general $U$, applying (3.15), we obtain through an integration by parts,

$$
\begin{aligned}
F(R) & -F(L)-s(U(R)-U(L))=\int_{L}^{R}\left[F^{\prime}(w)-s U^{\prime}(w)\right] d w \\
= & \int_{L}^{R}\left(f^{\prime}(w)-s\right) U^{\prime}(w) d w=\int_{L}^{R} U^{\prime}(w) d(f(w)-f[w ; L, R]) \\
= & \int_{L}^{R} U^{\prime \prime}(w)(f[w ; L, R]-f(w)) d w .
\end{aligned}
$$

The combination of this relation and (3.9) yields (3.16).

Definition 3.12. An ATW $\left\{x^{k}(t), y^{k}(t)\right\}$ of $\left\{u^{k}\right\}$ is called an asymptotic traveling discontinuity $(A T D)$ of $\left\{u^{k}\right\}$ if the ATW is essentially monotone in space. Namely,

(i) for each $k$, and for any integers $p$ and $q$ such that $I^{k}(t) \leq p<q \leq J^{k}(t)$, and for $0 \leq t \leq 1$,

$$
-\left(u_{q}^{k}(t)-u_{p}^{k}(t)\right) \operatorname{sign}(R-L) \leq \varepsilon_{k},
$$

and

(ii) for each $k$ and $t \in[0,1]$, if $j$ is an integer such that $I^{k}(t) \leq j \leq J^{k}(t)$ and that $\left(u_{j+1}^{k}(t)-u_{j}^{k}(t)\right)(R-L)<0$, then

$$
u_{j}^{k}(t), u_{j+1}^{k}(t) \in \mathcal{N}_{\varepsilon_{k}}(\{w: f(w)=f[w ; L, R]\}),
$$

where $\mathcal{N}_{\delta}(S)$ denotes the $\delta$-neighborhood of a set $S \in \mathbf{R}$. An ATD of $\left\{u^{k}\right\}$ is called an asymptotic traveling expansion shock (ATES) of $\left\{u^{k}\right\}$ if $(2.8)$ with $w_{-}=L$ and $w_{+}=R$ fails. In the last case we also say that $\left\{u^{k}\right\}$ harbors the ATES $\left\{x^{k}(t), y^{k}(t)\right\}$.

We are now ready to state our third WEI criterion for convergence.

Theorem 3.13. A semidiscrete scheme of the form (1.2)-(1.4) satisfying Assumption 3.3 converges if the scheme is unable to create a sequence of numerical solutions $\left\{u^{k}\right\}$ that harbors an ATES.

The proof of this theorem is contained in the Supplement to this paper.

We now turn our attention to convex conservation laws, i.e., the case $f^{\prime \prime}(w) \geq 0$.

We call a pair of numbers $\{L, R\}$ a rarefying pair if $L<R$ and $f[w ; L, R]>f(w)$ when $L<w<R$. We call a collection of data $\left\{v_{j}\right\}_{j=-p}^{n+p}$ a rarefying collection with respect to the pair $\{L, R\}$ if $L=v_{0} \leq v_{1} \leq \cdots \leq v_{n}=R$, and $L \leq v_{-1}$, and $R \geq v_{n+1}$. We let $\bar{g}_{j+1 / 2} \stackrel{\text { def }}{=} g\left(v_{j-p+1}, v_{j-p+2}, \ldots, v_{j+p}\right)$, where $g$ is the function (1.3) in its self-similar form. We add superscripts to denote a sequence of rarefying collections and the corresponding numerical fluxes.

Our fourth, and last, WEI criterion for convergence is as follows.

Theorem 3.14. A semidiscrete scheme of the form (1.2)-(1.4) satisfying Assumption 3.3 converges for convex conservation laws if, for any rarefying pair $\{L, R\}$, there is a constant $\delta>0$ such that the quadrature inequality

$$
\sum_{j=0}^{n-1}\left(v_{j+1}-v_{j}\right) \bar{g}_{j+1 / 2}+\delta<\int_{L}^{R} f[w ; L, R] d w
$$

holds for all rarefying collections $\left\{v_{j}\right\}_{j=-p}^{n+p}$ with respect to the pair $\{L, R\}$.

Proof. We prove the contrapositive. Hence, we assume the scheme does not converge. By Theorem 3.13, there exists a sequence of numerical solutions $\left\{u^{k}\right\}$ that harbors an ATES $\left\{x_{I^{k}(t)}^{k}, x_{J^{k}(t)}^{k}\right\}$. Since $f$ is convex, the two states $L$ and $R$ of 
the ATES form a rarefying pair $\{L, R\}$. The essentially monotone property of the ATES implies that, for each $k$ and each $t \in[0,1]$, there are two integers $l^{k}(t)$ and $r^{k}(t)$ with $I^{k}(t) \leq l^{k}(t)<r^{k}(t) \leq J^{k}(t)$ such that

$$
\begin{gathered}
L+\varepsilon_{k} \leq u_{l^{k}(t)+1}^{k}(t), \\
u_{r^{k}(t)-1}^{k}(t) \leq R-\varepsilon_{k}, \\
L-\varepsilon_{k}<u_{j}^{k}(t)<L+\varepsilon_{k} \quad \text { when } \quad I^{k}(t) \leq j \leq l^{k}(t), \\
R-\varepsilon_{k}<u_{j}^{k}(t)<R+\varepsilon_{k} \quad \text { when } \quad r^{k}(t) \leq j \leq J^{k}(t),
\end{gathered}
$$

and

$$
u_{j}^{k}(t) \leq u_{j+1}^{k}(t) \quad \text { when } \quad l^{k}(t) \leq j \leq r^{k}(t)-1 .
$$

Moreover, since $x_{I^{k}(t)}$ and $x_{J^{k}(t)}$ are $\varepsilon_{k^{-}}$-paths,

$$
u_{I^{k}(t)-1}^{k}(t)>u_{I^{k}(t)}^{k}(t)-\varepsilon_{k} \quad \text { if } \quad u_{I^{k}(t)+1}^{k}(t) \geq u_{I^{k}(t)}^{k}(t)+\varepsilon_{k},
$$

and

$$
u_{J^{k}(t)+1}^{k}(t)<u_{J^{k}(t)}^{k}(t)+\varepsilon_{k} \quad \text { if } \quad u_{J^{k}(t)-1}^{k}(t) \leq u_{J^{k}(t)}^{k}(t)-\varepsilon_{k} .
$$

For any fixed $t \in[0,1]$ and any positive integer $k$, construct the data $\left\{v_{j}\right\}_{j=-p}^{n+p}$ in the following way. Set $l^{\prime}=l^{k}(t)+1$ if $l^{k}(t)=I^{k}(t)$ and $u_{I^{k}(t)-1}^{k}(t) \leq u_{I^{k}(t)}^{k}(t)-\varepsilon_{k}$. Otherwise, set $l^{\prime}=l^{k}(t)$. Set $r^{\prime}=r^{k}(t)-1$ if $r^{k}(t)=J^{k}(t)$ and $u_{J^{k}(t)+1}^{k}(t) \geq$ $u_{J^{k}(t)}^{k}(t)+\varepsilon_{k}$. Otherwise, set $r^{\prime}=r^{k}(t)$.

Let $n=r^{\prime}-l^{\prime}$. Then set $v_{0}=L, v_{n}=R$, and $v_{j}=u_{l^{\prime}+j}^{k}(t)$ for $l^{\prime}+1 \leq j \leq r^{\prime}-1$. Also set

$$
v_{-1}= \begin{cases}L & \text { if } u_{l^{\prime}-1}^{k}(t)<L, \\ u_{l^{\prime}-1}^{k}(t) & \text { otherwise }\end{cases}
$$

and

$$
v_{n+1}= \begin{cases}R & \text { if } u_{r^{\prime}+1}^{k}(t)>R, \\ u_{r^{\prime}+1}^{k}(t) & \text { otherwise, }\end{cases}
$$

Finally, set $v_{j}=u_{l^{\prime}+j}^{k}(t)$ for $-p \leq j \leq-2$ and for $n+2 \leq j \leq n+p$. In the notations $\left\{v_{j}\right\}_{j=-p}^{n+p}$, and later in $\bar{g}_{j+\frac{1}{2}}$, we ignore the apparent dependence on $k$, as this will not be harmful to the proof. It is straightforward to verify that the data $\left\{v_{j}\right\}_{j=-p}^{n+p}$ form a rarefying collection with respect to the rarefying pair $\{L, R\}$. Moreover, by (3.20), (3.21), (3.23) and (3.24), the construction also implies that $\left|v_{j}-u_{l^{\prime}+j}^{k}(t)\right|<2 \varepsilon_{k}$ when $-p \leq j \leq n+p$, and, in particular, $v_{j}=u_{l^{\prime}+j}^{k}(t)$ when $j \neq-1,0, n, n+1$.

With the relation (3.7) applied to (3.8), the limit in Corollary 3.11 becomes

$$
\begin{aligned}
\int_{0}^{1} \sum_{j=I^{k}(t)}^{J^{k}(t)-1} \int_{u_{j}^{k}(t)}^{u_{j+1}^{k}(t)} U^{\prime \prime}(w)\left(g_{j+\frac{1}{2}}(t)-f(w)\right) d w \phi_{j}(t) d t \\
\\
\rightarrow \int_{L}^{R} U^{\prime \prime}(w)(f[w ; L, R]-f(w)) d w \int_{x=s t} \phi(x, t) d t
\end{aligned}
$$


It is known that for convex problems, the entropy condition for $U(w)=w^{2} / 2$ is sufficient for convergence. For this $U(w),(3.27)$ becomes

$$
\begin{aligned}
\int_{0}^{1} \sum_{j=I^{k}(t)}^{J^{k}(t)-1} \int_{u_{j}^{k}(t)}^{u_{j+1}^{k}(t)}\left(g_{j+\frac{1}{2}}(t)-f(w)\right) d w \phi_{j}(t) d t \\
\quad \rightarrow \int_{L}^{R}(f[w ; L, R]-f(w)) d w \int_{x=s t} \phi(x, t) d t
\end{aligned}
$$

Since $\phi(x, t)$ is smooth with compact support, since the total spatial variations of $u^{k}(x, t)$ have a uniform bound $B$, and since $\left|x_{I^{k}(t)}^{k}-s t\right|+\left|x_{J^{k}(t)}^{k}-s t\right|<\varepsilon_{k}$, we have

$$
\left|\int_{0}^{1} \sum_{j=I^{k}(t)}^{J^{k}(t)-1} \int_{u_{j}^{k}(t)}^{u_{j+1}^{k}(t)}\left(g_{j+\frac{1}{2}}(t)-f(w)\right) d w\left(\phi(s t, t)-\phi_{j}(t)\right) d t\right|<C \varepsilon_{k},
$$

where $C$ is independent of $k$.

Claim. For the data $\left\{v_{j}\right\}_{j=-p}^{n+p}$,

$$
\left|\sum_{j=I^{k}(t)}^{J^{k}(t)-1} \int_{u_{j}^{k}(t)}^{u_{j+1}^{k}(t)}\left(g_{j+\frac{1}{2}}(t)-f(w)\right) d w-\sum_{i=0}^{n-1} \int_{v_{i}}^{v_{i+1}}\left(\bar{g}_{i+\frac{1}{2}}-f(w)\right) d w\right| \leq C \varepsilon_{k},
$$

where $\bar{g}_{i+\frac{1}{2}}=g\left(v_{j-p+1}, v_{j-p+2}, \ldots, v_{j+p}\right)$ and $g$ is the function in (1.3) in its selfsimilar form.

In fact,

$$
\begin{aligned}
& \left|\sum_{j=I^{k}(t)}^{J^{k}(t)-1} \int_{u_{j}^{k}(t)}^{u_{j+1}^{k}(t)}\left(g_{j+\frac{1}{2}}(t)-f(w)\right) d w-\sum_{i=0}^{n-1} \int_{v_{i}}^{v_{i+1}}\left(\bar{g}_{i+\frac{1}{2}}-f(w)\right) d w\right| \\
& \leq\left|\sum_{j=I^{k}(t)}^{i^{\prime}-1} \int_{u_{j}^{k}(t)}^{u_{j+1}^{k}(t)}\left(g_{j+\frac{1}{2}}(t)-f(w)\right) d w\right|+\left|\sum_{j=j^{\prime}}^{J^{k}(t)-1} \int_{u_{j}^{k}(t)}^{u_{j+1}^{k}(t)}\left(g_{j+\frac{1}{2}}(t)-f(w)\right) d w\right| \\
& \quad+\left|\sum_{i=0}^{n-1}\left[\int_{u_{i^{\prime}+i}^{k}(t)}^{u_{i^{\prime}+i+1}^{k}(t)}\left(g_{i^{\prime}+i+\frac{1}{2}}(t)-f(w)\right) d w-\int_{v_{i}}^{v_{i+1}}\left(\bar{g}_{i+\frac{1}{2}}-f(w)\right) d w\right]\right| \\
& =A_{1}+A_{2}+A_{3} .
\end{aligned}
$$

If $A_{1}$ contains only one term, then $\left|u_{I^{k}(t)+1}^{k}(t)-u_{I^{k}(t)}^{k}(t)\right|<2 \varepsilon_{k}$. Therefore, $A_{1}<$ $C \varepsilon_{k}$. If $A_{1}$ contains more than one term, then in each term of $A_{1},\left|u_{j+1}^{k}(t)-u_{j}^{k}(t)\right|<$ $2 \varepsilon_{k}$. Hence, by Corollary 3.4 (ii), $\left|g_{j+\frac{1}{2}}(t)-f\left(u_{j}^{k}(t)\right)\right|<C \varepsilon_{k}$ holds. Therefore, $A_{1}<$ $C \varepsilon_{k}$ holds in all cases, where $C$ is again independent of $k$. Similarly, $A_{2}<C \varepsilon_{k}$. To prove $A_{3}<C \varepsilon_{k}$, it suffices to notice that, among the $n$ terms in the summation, there are at most $2 p$ nonzero terms, and that the absolute value of each term is bounded by $C \varepsilon_{k}$. Hence, the claim is true. 
Let $\phi(x, t) \geq 0$, and $\int_{x=s t} \phi(x, t) d t>0$. Combining (3.17), (3.29) and (3.30), and using the triangle inequality, we arrive at

$$
\begin{aligned}
& \varlimsup_{k \rightarrow \infty} \int_{0}^{1} \sum_{j=I^{k}(t)}^{J^{k}(t)-1} \int_{u_{j}^{k}(t)}^{u_{j+1}^{k}(t)}\left(g_{j+\frac{1}{2}}(t)-f(w)\right) d w \phi_{j}(t) d t \\
& \quad \leq\left[\int_{L}^{R}(f[w ; L, R]-f(w)) d w-\delta\right] \int_{x=s t} \phi(x, t) d t .
\end{aligned}
$$

This contradicts (3.28), and proves the theorem.

\section{Applications}

In this section we apply Theorem 3.14 to two classes of schemes for convex problems. The building blocks for both classes are the so-called E-schemes [17]. Let $g^{E}(\cdot, \cdot)$ be the flux of any E-scheme, i.e., it is Lipschitz continuous, and satisfies

$$
\operatorname{sgn}\left(w_{j+1}-w_{j}\right)\left(g^{E}\left(w_{j}, w_{j+1}\right)-f(w)\right) \leq 0
$$

for all $w$ between $w_{j}$ and $w_{j+1}$. We now turn to our first example.

Example 4.1. Consider the class of generalized MUSCL schemes

$$
\frac{d}{d t} u_{j}=-\frac{1}{h} \Delta_{+} g^{E}\left(u_{j-1}+s_{j-1} h / 2, u_{j}-s_{j} h / 2\right) .
$$

In [18] Osher proposed and analyzed this class with the CEI approach. A nonlinear slope limiter was introduced to enforce a cell entropy inequality for $u_{j+1}<u_{j}$. Let $\tilde{u}_{j+\frac{1}{2}}$ satisfy

$$
\int_{u_{j}}^{u_{j+1}} f^{\prime}(w)\left(w-\tilde{u}_{j+\frac{1}{2}}\right) d w=0 .
$$

Osher [18] proved that a generalized MUSCL scheme (4.2) for the convex problem (1.1) converges provided that for each $j$,

$$
0 \leq \frac{h s_{j}}{\Delta_{+} u_{j}}, \frac{h s_{j}}{\Delta_{-} u_{j}} \leq 1
$$

and that if $u_{j}>u_{j+1}$, the nonlinear slope limiter

$$
-h s_{j} \leq 2 \max \left(\min \left(\left(u_{j}-\tilde{u}_{j+\frac{1}{2}}\right),\left(\tilde{u}_{j-\frac{1}{2}}-u_{j}\right)\right), 0\right)
$$

holds.

With WEI, we are able to prove the convergence without using the nonlinear slope limiter (4.4). In fact we can do even better by proving the following result.

Theorem 4.2. A generalized MUSCL scheme with an E-scheme building block converges provided that the slope $s_{j}$ satisfies: $s_{j}=0$ if $u_{j}$ is an extremum;

$$
0 \leq \frac{h s_{j}}{\Delta_{+} u_{j}}, \frac{h s_{j}}{\Delta_{-} u_{j}} \leq 2
$$

if $u_{j-1}$ is a maximum or $u_{j+1}$ is a minimum; and (4.3) holds if $u_{j}<u_{j+1}$.

Proof. Tadmor [25] shows that the numerical solution satisfying the conditions of the theorem obeys Assumption 3.3. Hence, the WEI criterion is applicable. Let $\{L, R\}$ be a rarefying pair and the data $\left\{v_{j}\right\}_{j=-p}^{n+p}$ be a rarefying collection with respect to the pair. Then Osher's cell entropy inequality $\int_{v_{j}}^{v_{j+1}}\left(\bar{g}_{j+1 / 2}-f(w)\right) d w \leq$ 
0 holds for $0 \leq j \leq n-1$ since $v_{j} \leq v_{j+1}$, which implies $\sum_{j=0}^{n-1}\left(v_{j+1}-v_{j}\right) \bar{g}_{j+1 / 2} \leq$ $\int_{L}^{R} f(w) d w$ (see [18]). Hence, the inequality (3.17) holds for any positive constant $\delta<\int_{L}^{R}(f[w ; L, R]-f(w)) d w$, and such a $\delta$ does exist because $\{L, R\}$ is a rarefying pair. This proves the theorem.

Example 4.3. In [24] Sweby investigated a large class of high-resolution schemes using flux limiters. Here we consider the semidiscrete version of these schemes. The building block is again an arbitrary E-scheme (4.1). We borrow the following notations from [24]:

$$
\begin{gathered}
\left(\Delta f_{j+\frac{1}{2}}\right)^{+}=f\left(u_{j+1}\right)-g^{E}\left(u_{j}, u_{j+1}\right), \\
\left(\Delta f_{j+\frac{1}{2}}\right)^{-}=f\left(u_{j}\right)-g^{E}\left(u_{j}, u_{j+1}\right), \\
r_{j}^{+}=\left(\Delta f_{j-\frac{1}{2}}\right)^{+} /\left(\Delta f_{j+\frac{1}{2}}\right)^{+}, \quad r_{j}^{-}=\left(\Delta f_{j+\frac{1}{2}}\right)^{-} /\left(\Delta f_{j-\frac{1}{2}}\right)^{-} .
\end{gathered}
$$

We also set

$$
\left(D f_{j+\frac{1}{2}}\right)^{ \pm}=\left(\Delta f_{j+\frac{1}{2}}\right)^{ \pm} / \Delta_{+} u_{j}
$$

The numerical flux of the semidiscrete version of the schemes with flux limiters has the following form:

$$
g_{j+\frac{1}{2}}=g^{E}\left(u_{j}, u_{j+1}\right)+\frac{1}{2} \psi\left(r_{j}^{+}\right)\left(\Delta f_{j+\frac{1}{2}}\right)^{+}+\frac{1}{2} \psi\left(r_{j+1}^{-}\right)\left(\Delta f_{j+\frac{1}{2}}\right)^{-} .
$$

Here the function $\psi$ defines the flux limiter of the scheme. Sweby [24] identified the class of functions $\psi$ for the scheme (4.6) to be second-order accurate away from nonsonic extrema and TVD. The convergence of these schemes has been an open problem owing to the subtler issue of entropy consistency.

Consider the following Chakravarthy and Osher limiters:

$$
\psi_{c}(r)= \begin{cases}0, & r<0, \\ r, & 0 \leq r<c, \\ c, & r \geq c .\end{cases}
$$

In the special case $c=1$, by adding artificial compression/rarefaction (ACR), and using CEI, Osher and Chakravarthy [19] were able to prove the convergence of the modified schemes for the convex problem (1.1). In the following, by applying the WEI criterion, we first show that without the addition of ACR, the schemes still converge.

Theorem 4.4. The numerical solutions of the schemes (1.2)-(1.4) converge for the convex problem (1.1) provided that the numerical flux $g_{j+\frac{1}{2}}$ satisfies:

(i) $g_{j+\frac{1}{2}} \geq f\left(u_{j}\right)$ if $u_{j}-u_{j \pm 1} \geq 0$, and $g_{j-\frac{1}{2}} \geq f\left(u_{j}\right)$ if $u_{j}-u_{j \pm 1} \leq 0$.

(ii) When $u_{j+1}>u_{j}, g_{j+\frac{1}{2}}$ is defined by (4.6) where $g^{E}$ is the numerical flux function of any E-scheme, and $\psi(r)=\psi_{1}(r)$ is defined by (4.7).

Proof. Let $u_{j+1}$ be a spatial maximum; thus $r_{j+1}^{-} \leq 0$. Hence, it follows from (4.7) that $\psi\left(r_{j+1}^{-}\right)=0$. Therefore

$$
g_{j+\frac{1}{2}}=g^{E}\left(u_{j}, u_{j+1}\right)+\frac{1}{2} \psi\left(r_{j}^{+}\right)\left(\Delta f_{j+\frac{1}{2}}\right)^{+} \leq g^{E}\left(u_{j}, u_{j+1}\right)+\left(\Delta f_{j+\frac{1}{2}}\right)^{+}=f\left(u_{j+1}\right) .
$$

Replacing $j$ with $j-1$ in the preceding inequality, we see that if $u_{j}-u_{j \pm 1} \geq 0$, then $g_{j-\frac{1}{2}} \leq f\left(u_{j}\right)$. Similarly, if $u_{j}-u_{j \pm 1} \leq 0$, then $g_{j+\frac{1}{2}} \leq f\left(u_{j}\right)$. These two inequalities and condition (i) of the theorem verify Assumption 3.3. Next, let $\{L, R\}$ 
be an arbitrary rarefying pair. Using Theorem 3.14 to prove the convergence of the scheme, we need to show that there exists a $\delta>0$ such that for any rarefying collection $\left\{v_{j}\right\}_{j=-p}^{n+p}$ with respect to the pair $\{L, R\}$, the inequality (3.17) holds.

For convenience, define

$$
\bar{f}[c, d] \stackrel{\text { def }}{=} \int_{c}^{d}\{f[w ; c, d]-f(w)\} d w,
$$

and

$$
S_{j+\frac{1}{2}} \stackrel{\text { def }}{=} \int_{v_{j}}^{v_{j+1}}\left[\bar{g}_{j+\frac{1}{2}}-f(w)\right] d w
$$

Since $f$ is convex,

$$
\bar{f}[a, b]+\bar{f}[b, c] \leq \bar{f}[a, c] \quad \text { if } \quad a \leq b \leq c .
$$

Applying the facts that $\psi(r) \leq 1$ and $\bar{g}^{E}\left(v_{j}, v_{j+1}\right) \leq \min \left(f\left(v_{j}\right), f\left(v_{j+1}\right)\right)$ to (4.6), we get

$$
S_{j+\frac{1}{2}} \leq \bar{f}\left[v_{j}, v_{j+1}\right] \quad \text { if } \quad 0 \leq j \leq n-1 .
$$

Summing over (4.11) and using (4.10) and the fact that $\left\{v_{j}\right\}_{j=-p}^{n+p}$ is a rarefying collection with respect to the pair $\{L, R\}$, we obtain that, for $1 \leq i \leq n-1$,

$$
\sum_{j=0}^{n-1} S_{j+\frac{1}{2}} \leq \sum_{j=0}^{n-1} \bar{f}\left[v_{j}, v_{j+1}\right] \leq \bar{f}\left[L, v_{i}\right]+\bar{f}\left[v_{i}, R\right] \leq \bar{f}[L, R] .
$$

We assume that no $\delta>0$ exists with the aforementioned property. Then there exists a sequence of rarefying collections $\left\{v_{j}^{\nu}, j=-p,-p+1, \ldots, n^{\nu}+p\right\}_{\nu=1}^{\infty}$ with respect to the pair $\{L, R\}$ such that

$$
\lim _{\nu \rightarrow \infty} \sum_{j=0}^{n^{\nu}-1} S_{j+\frac{1}{2}}^{\nu}=\lim _{\nu \rightarrow \infty} \sum_{j=0}^{n^{\nu}-1} \bar{f}\left[v_{j}^{\nu}, v_{j+1}^{\nu}\right]=\bar{f}[L, R] .
$$

Claim. The inequality (4.13) implies that there exists a sequence of integers $\left\{j^{\nu}\right\}$ satisfying $0 \leq j^{\nu} \leq n^{\nu}-1$ such that

$$
\lim _{\nu \rightarrow \infty} v_{j^{\nu}}^{\nu}=L \quad \text { and } \quad \lim _{\nu \rightarrow \infty} v_{j^{\nu}+1}^{\nu}=R .
$$

For, otherwise, there exists a constant $\rho>0$, a subsequence of the rarefying collections, still denoted by $\left\{v_{j}^{\nu}, j=-p,-p+1, \ldots, n^{\nu}+p\right\}_{\nu=1}^{\infty}$, and a sequence of integers $\left\{i^{\nu}\right\}$ satisfying $1 \leq i^{\nu} \leq n^{\nu}-1$ such that $L+\rho<v_{i^{\nu}}^{\nu}<R-\rho$. The convexity of $f$, the definition of rarefying pairs, and (4.12) imply

$$
\begin{aligned}
& \sum_{j=0}^{n^{\nu}-1} \bar{f}\left[v_{j}^{\nu}, v_{j+1}^{\nu}\right] \leq \bar{f}\left[L, v_{i^{\nu}}^{\nu}\right]+\bar{f}\left[v_{i^{\nu}}^{\nu}, R\right] \\
& \quad \leq H_{\rho} \stackrel{\text { def }}{=} \max (\bar{f}[L, L+\rho]+\bar{f}[L+\rho, R], \bar{f}[L, R-\rho]+\bar{f}[R-\rho, R]) \\
& \quad<\bar{f}[L, R] .
\end{aligned}
$$

This contradicts (4.13) because $H_{\rho}$ is independent of $\nu$. Hence, the claim is true. 
On the one hand, combining (4.10), (4.11), (4.13) and (4.14), we obtain

$$
\begin{aligned}
\bar{f}[L, R] & =\lim _{\nu \rightarrow \infty} \sum_{j=0}^{n^{\nu}-1} S_{j+\frac{1}{2}}^{\nu}=\lim _{\nu \rightarrow \infty}\left(\sum_{j=0}^{j^{\nu}-1} S_{j+\frac{1}{2}}^{\nu}+S_{j^{\nu}+\frac{1}{2}}^{\nu}+\sum_{j=j^{\nu}+1}^{n^{\nu}-1} S_{j+\frac{1}{2}}^{\nu}\right) \\
& \leq \lim _{\nu \rightarrow \infty}\left(\bar{f}\left[L, v_{j^{\nu}}^{\nu}\right]+S_{j^{\nu}+\frac{1}{2}}^{\nu}+\bar{f}\left[v_{j^{\nu}+1}^{\nu}, R\right]\right)=\lim _{\nu \rightarrow \infty} S_{j^{\nu}+\frac{1}{2}}^{\nu} \leq \bar{f}[L, R] .
\end{aligned}
$$

This implies

$$
\lim _{\nu \rightarrow \infty} S_{j^{\nu}+\frac{1}{2}}^{\nu}=\bar{f}[L, R] .
$$

On the other hand, applying (4.14) and the definition of the rarefying collections with respect to the rarefying pair $\{L, R\}$ to (4.6), we get $\lim _{\nu \rightarrow \infty} \bar{g}_{j^{\nu}+\frac{1}{2}}^{\nu}=g^{E}(L, R)$, and hence $\lim _{\nu \rightarrow \infty} S_{j^{\nu}+\frac{1}{2}}^{\nu} \leq 0$. This contradicts (4.17) since $\bar{f}[L, R]>0$ by the definition of the rarefying pair $\{L, R\}$.

When the building blocks of the high-resolution schemes are such well-known monotone schemes as the Godunov, the Engquist-Osher or Lax-Friedrichs schemes, we can do even better. The following give the numerical fluxes of these three schemes:

(i) The Godunov scheme:

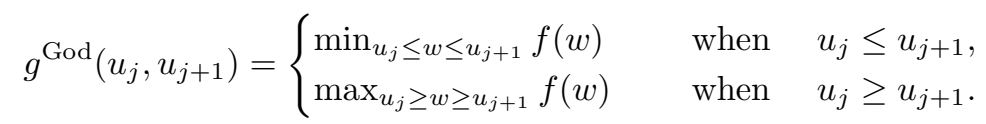

(ii) The Engquist-Osher scheme:

$$
g^{\mathrm{EO}}\left(u_{j}, u_{j+1}\right)=\int_{0}^{u_{j}} \max \left(f^{\prime}(w), 0\right) d w+\int_{0}^{u_{j+1}} \min \left(f^{\prime}(w), 0\right) d w+f(0) .
$$

(iii) The Lax-Friedrichs scheme:

$$
g^{\mathrm{LF}}\left(u_{j}, u_{j+1}\right)=\frac{f\left(u_{j}\right)+f\left(u_{j+1}\right)}{2}-\frac{a}{2}\left(u_{j+1}-u_{j}\right)
$$

where $a \geq \max \left|f^{\prime}(w)\right|$.

When we use these three monotone schemes as the building blocks, we have the following result.

Theorem 4.5. The numerical solutions of the schemes (1.2)-(1.4) converge for the convex problem (1.1) provided that the numerical flux $g_{j+\frac{1}{2}}$ satisfies:

(i) $g_{j+\frac{1}{2}} \geq f\left(u_{j}\right)$ if $u_{j}-u_{j \pm 1} \geq 0$, and $g_{j-\frac{1}{2}} \geq f\left(u_{j}\right)$ if $u_{j}-u_{j \pm 1} \leq 0$.

(ii) When $u_{j+1}>u_{j}, g_{j+\frac{1}{2}}$ is defined by (4.6) where $\psi(r)=\psi_{c}(r)$ is given by (4.7) with $1 \leq c \leq 2$, provided that $g^{E}(\cdot, \cdot)$ is the numerical flux of one of the aforementioned monotone schemes.

Proof. Let $\{L, R\}$ be a rarefying pair. Consider any rarefying collection $\left\{v_{j}\right\}_{j=-p}^{n+p}$ with respect to this pair. Following the arguments of the proof of Theorem 4.4, we only need to show that although (4.11) may now be false, (4.12) is still true. For convenience, we keep the same notations $\left(\Delta f_{j+\frac{1}{2}}\right)^{ \pm},\left(D f_{j+\frac{1}{2}}\right)^{ \pm}$and $r_{j}^{ \pm}$when $u$ is replaced by $v$. 
Using $\psi_{c}\left(r_{0}^{+}\right)=\psi_{c}\left(r_{n}^{-}\right)=0$, we have

$$
\begin{aligned}
\sum_{j=0}^{n-1}\left(S_{j+}\right. & -\overline{2} \\
= & -\frac{1}{2}\left\{\left(v_{j}, v_{j+1}\right]\right) \\
& -\frac{1}{2} \sum_{j=1}^{n-2}\left\{\left[1-\psi_{c}\left(r_{j}^{+}\right)\right]\left(\Delta f_{j+\frac{1}{2}}\right)^{+}+\left[1-\psi_{c}\left(r_{j+1}^{-}\right)\right]\left(\Delta_{j+\frac{1}{2}}\right)^{-}\right\} \Delta_{+} v_{j} \\
& -\frac{1}{2}\left\{\left[1-\psi_{c}\left(r_{n-1}^{+}\right)\right]\left(\Delta f_{n-\frac{1}{2}}\right)^{+}+\left(\Delta f_{n-\frac{1}{2}}\right)^{-}\right\} \Delta_{+} v_{n-1} \\
\leq & -\frac{1}{2}\left[\left(\left(D f_{\frac{1}{2}}\right)^{+}+\left(D f_{\frac{1}{2}}\right)^{-}\right)\left(\Delta_{+} v_{0}\right)^{2}-\left(D f_{\frac{3}{2}}\right)^{-}\left(\Delta_{+} v_{0}\right)\left(\Delta_{+} v_{1}\right)\right] \\
& -\frac{1}{2} \sum_{j=1}^{n-2}\left[\left(D f_{j+\frac{1}{2}}\right)^{+}\left(\Delta_{+} v_{j}\right)^{2}-\left(D f_{j-\frac{1}{2}}\right)^{+}\left(\Delta_{+} v_{j}\right)\left(\Delta_{+} v_{j-1}\right)\right. \\
& \left.+\left(D f_{j+\frac{1}{2}}\right)^{-}\left(\Delta_{+} v_{j}\right)^{2}-\left(D f_{j+\frac{3}{2}}\right)^{-}\left(\Delta_{+} v_{j}\right)\left(\Delta_{+} v_{j+1}\right)\right] \\
& -\frac{1}{2}\left[\left(\left(D f_{n-\frac{1}{2}}\right)^{+}+\left(D f_{n-\frac{1}{2}}\right)^{-}\right)\left(\Delta_{+} v_{n-1}\right)^{2}-\left(D f_{n-\frac{3}{2}}\right)^{+}\left(\Delta_{+} v_{n-1}\right)\left(\Delta_{+} v_{n-2}\right)\right] \\
\leq & -\frac{1}{2}\left\{\left[\left(D f_{\frac{1}{2}}\right)^{+}+\left(D f_{\frac{1}{2}}\right)^{-}\right]\left(\Delta_{+} v_{0}\right)^{2}-\frac{1}{2}\left(D f_{\frac{3}{2}}\right)^{-}\left[\left(\Delta_{+} v_{0}\right)^{2}+\left(\Delta_{+} v_{1}\right)^{2}\right]\right\} \\
& -\frac{1}{2} \sum_{j=1}^{n-2}\left\{\left(D f_{j+\frac{1}{2}}\right)^{+}\left(\Delta_{+} v_{j}\right)^{2}-\frac{1}{2}\left(D f_{j-\frac{1}{2}}\right)^{+}\left[\left(\Delta_{+} v_{j}\right)^{2}+\left(\Delta_{+} v_{j-1}\right)^{2}\right]\right. \\
& +\frac{1}{4}\left(D f_{\frac{1}{2}}\right)^{+}\left(\Delta_{+} v_{0}\right)^{2}-\frac{1}{4} \sum_{j=1}^{n-1}\left[\Delta_{+}\left(D f_{j-\frac{1}{2}}\right)^{+}\right]\left(\Delta_{+} v_{j}\right)^{2} \\
& -\frac{1}{4}\left(D f_{n-\frac{1}{2}}\right)^{+}\left(\Delta_{+} v_{n-1}\right)^{2}-\frac{1}{4}\left(D f_{\frac{1}{2}}\right)^{-}\left(\Delta_{+} v_{0}\right)^{2} \\
& \left.-\frac{1}{2}\left\{\left[\left(D f_{n-\frac{1}{2}}\right)^{-}\left(\Delta_{+}\right)_{j}\right)^{2}-\frac{1}{2}\left(D f_{j+\frac{3}{2}}\right)^{-}\left[\left(\Delta_{+} v_{j}\right)^{2}+\left(D f_{j+\frac{1}{2}}\right)^{-}\right]\left(\Delta_{+} v_{j+1}\right)^{2}\right]\right\} \\
& \left.-\left(D f_{n-\frac{1}{2}}\right)^{-}\right]\left(\Delta_{+} v_{n-1}\right)^{2}-\frac{1}{4}\left(D f_{n-\frac{1}{2}}\right)^{-}\left(\Delta_{+} v_{n-1}\right)^{2} . \\
& \left.\left.=\Delta_{n-1}\right)^{2}+\left(\Delta_{+} v_{n-2}\right)^{2}\right\} \\
& \\
& \\
&
\end{aligned}
$$

Here we have used the condition $f_{c}(r) \leq r$, the fact that $\left(D f_{j+\frac{1}{2}}\right)^{ \pm} \geq 0$, and the elementary inequality $a b \leq\left(a^{2}+b^{2}\right) / 2$.

First if the building block is the Lax-Friedrichs scheme, we have $\left(D f_{j+\frac{1}{2}}\right)^{ \pm}=$ $\left(a \pm D_{+} f\left(v_{j}\right)\right) / 2$. Hence,

$$
\begin{gathered}
\Delta_{+}\left(D f_{j-\frac{1}{2}}\right)^{+}=\Delta_{-}\left(D_{+} f\left(v_{j}\right)\right) \geq 0 \quad \text { for } \quad 1 \leq j \leq n-1, \quad \text { and } \\
\Delta_{+}\left(D f_{j+\frac{1}{2}}\right)^{-}=-\Delta_{+}\left(D_{+} f\left(v_{j}\right)\right) \leq 0 \quad \text { for } \quad 0 \leq j \leq n-2
\end{gathered}
$$


since $f$ is convex. Applying these two inequalities to (4.18) and noticing that $\left(D f_{j+\frac{1}{2}}\right)^{ \pm} \geq 0$ for $0 \leq j \leq n-1$, we get the desired inequality (4.12).

Next suppose the building block is the Godunov scheme or the Engquist-Osher scheme. Then we have

$$
g^{\mathrm{God}}\left(v_{j}, v_{j+1}\right)=g^{\mathrm{EO}}\left(v_{j}, v_{j+1}\right)=\min _{v_{j} \leq w \leq v_{j+1}} f(w),
$$

when $v_{j+1} \geq v_{j}$, as is the case for $0 \leq j \leq n-1$. Hence, for $0 \leq j \leq n-1$,

$$
\begin{gathered}
\left(\Delta f_{j+\frac{1}{2}}\right)^{+}=0 \quad \text { and } \quad\left(\Delta f_{j+\frac{1}{2}}\right)^{-}=-\Delta_{+} f\left(v_{j}\right) \quad \text { when } \quad f^{\prime}\left(v_{j+1}\right) \leq 0 \\
\left(\Delta f_{j+\frac{1}{2}}\right)^{-}=0 \quad \text { and } \quad\left(\Delta f_{j+\frac{1}{2}}\right)^{+}=\Delta_{+} f\left(v_{j}\right) \quad \text { when } \quad f^{\prime}\left(v_{j}\right) \geq 0
\end{gathered}
$$

and

$$
\left(\Delta f_{j+\frac{1}{2}}\right)^{-}=f\left(v_{j}\right)-f\left(w_{\min }\right) \quad \text { and } \quad\left(\Delta f_{j+\frac{1}{2}}\right)^{+}=f\left(v_{j+1}\right)-f\left(w_{\min }\right)
$$

when $f^{\prime}\left(v_{j}\right) \leq 0 \geq f^{\prime}\left(v_{j+1}\right)$.

There is a slight difference in the arguments according to the different locations of the sonic point. We just consider the case in which there is an integer $p, 1 \leq p \leq$ $n-2$, such that $f^{\prime}\left(v_{p}\right) \leq 0 \leq f^{\prime}\left(v_{p+1}\right)$, for the proof in this case contains all the essential arguments for other cases. Applying (4.20), (4.21) and (4.22) to (4.18), we have

$$
\begin{aligned}
\sum_{j=0}^{n-1}( & \left.S_{j+\frac{1}{2}}-\bar{f}\left[v_{j}, v_{j+1}\right]\right) \\
& =-\frac{1}{4}\left(D f_{\frac{1}{2}}\right)^{-}\left(\Delta_{+} v_{0}\right)^{2}-\frac{1}{4} \sum_{j=0}^{p-1}\left[\left(D f_{j+\frac{1}{2}}\right)^{-}-\left(D f_{j+\frac{3}{2}}\right)^{-}\right]\left(\Delta_{+} v_{j}\right)^{2} \\
& -\frac{1}{4}\left[\left(D f_{p+\frac{1}{2}}\right)^{+}+\left(D f_{p+\frac{1}{2}}\right)^{-}\right]\left(\Delta_{+} v_{p}\right)^{2} \\
& -\frac{1}{4} \sum_{j=p+1}^{n-1}\left[\left(D f_{j+\frac{1}{2}}\right)^{+}-\left(D f_{j-\frac{1}{2}}\right)^{+}\right]\left(\Delta_{+} v_{j}\right)^{2}-\frac{1}{4}\left(D f_{n-\frac{1}{2}}\right)^{+}\left(\Delta_{+} v_{n-1}\right)^{2} .
\end{aligned}
$$

To verify (4.12), it suffices to show that every term in (4.23) is less than or equal to zero. In fact when $j \leq p-1,\left(D f_{j+\frac{1}{2}}\right)^{-}=-D_{+} f\left(v_{j}\right)$; when $j \geq p+1,\left(D f_{j+\frac{1}{2}}\right)^{+}=$ $D_{+} f\left(v_{j}\right)$. The convexity of $f$ then implies that $\left(D f_{j+\frac{1}{2}}\right)^{-} \geq\left(D f_{j+\frac{3}{2}}\right)^{-}$holds when $0 \leq j \leq p-2$, and $\left(D f_{j+\frac{1}{2}}\right)^{+} \geq\left(D f_{j-\frac{1}{2}}\right)^{+}$holds when $p+2 \leq j \leq n-1$. It remains to show that

$$
\left(D f_{p-\frac{1}{2}}\right)^{-} \geq\left(D f_{p+\frac{1}{2}}\right)^{-} \quad \text { and } \quad\left(D f_{p+\frac{1}{2}}\right)^{+} \leq\left(D f_{p+\frac{3}{2}}\right)^{+} .
$$

Actually, the convexity of $f$ implies that

$$
\left(D f_{p+\frac{1}{2}}\right)^{-}=\frac{f\left(v_{p}\right)-f\left(v_{\min }\right)}{\Delta_{+} v_{p}} \leq \frac{f\left(v_{p}\right)-f\left(v_{\min }\right)}{v_{\min }-v_{p}} \leq\left(D f_{p-\frac{1}{2}}\right)^{-} .
$$

This is the first inequality in (4.24). The second can be proved similarly. We have thus proved the convergence when the building block is either the Godunov scheme or the Engquist-Osher scheme. 


\section{ACKNOWLEDGEMENT}

The author is indebted to Professor Stanley Osher for suggesting this problem and for his advice and encouragement. Thanks are due also to Professors Chi-Wang Shu, Bernardo Cockburn, Weinan E, Guiqiang Chen and Jianguo Liu for many valuable discussions. The author is also very grateful to the referees who carefully read the paper and with their suggestions significantly improved the presentation of the paper.

Essential work of this paper was conducted when the author was visiting the School of Mathematics, University of Minnesota at Minneapolis. The author expresses thanks for their hospitality. The author also thanks the IMA of the University of Minnesota for including an early version [29] of this paper in the IMA preprint series.

\section{REFERENCES}

1. F. Bouchut, C. Bourdarias, and B. Perthame, Un exemple de méthode MUSCL satisfaisant toutes les inégalités d'entropie numériques, C.R. Acad. Sci. Paris, Sér. I. Math. 317 (1993), 619-624. MR 94e:65119

2. G. Chen and J. Liu, Convergence of second-order schemes for isentropic gas dynamics, Math. Comp. 61 (1993), 607-629. MR 94a:65047

3. B. Cockburn, F. Coquel, and F. LeFloch, An error estimate for finite volume methods for multidimensional conservation laws, Math. Comp. 63 (1994), 77-103. MR 95d:65078

4. B. Cockburn and P. Gremaud, An error estimate for finite element methods for scalar conservation laws, SIAM J. Numer. Anal. (to appear).

5. F. Coquel and F. LeFloch, Convergence of finite difference schemes for conservation laws in several space dimensions: the corrected antidiffusive flux approach, Math. Comp. 57 (1991), 169-210. MR 91m:65229

6. M. Crandall and A. Majda, Monotone difference approximations for scalar conservation laws, Math. Comp. 34 (1980), 1-21. MR 81b:65079

7. R. J. DiPerna, Convergence of approximate solutions to conservation laws, Arch. Rational Mech. Anal. 82 (1983), 27-70. MR 84k:35091

8. S. K. Godunov, A finite difference method for the numerical computation of discontinuous solutions of the equations of fluid dynamics, Mat. Sb. 47 (1959), 271-306. MR 22:10194

9. J. Goodman and R. LeVeque, On the accuracy of stable schemes for $2 D$ scalar conservation laws, Math. Comp. 45 (1985), 15-21. MR 86f:65149

10. A. Harten, J. M. Hyman, and P. D. Lax, On finite-difference approximations and entropy conditions for shocks, Comm. Pure Appl. Math. 29 (1976), 297-322. MR 54:1640

11. G. Jennings, Discrete shocks, Comm. Pure Appl. Math. 27 (1974), 25-37. MR 49:3358

12. C. Johnson, A. Szepessy, and P. Hansbo, On the convergence of shock-capturing streamline diffusion finite element methods for hyperbolic conservation laws, Math. Comp. 54 (1990), 107-129. MR 90j:65118

13. S. N. Kružkov, First order quasilinear equations in several independent variables, Math. USSR Sb. 47 (1970), 217-243. MR 42:2159

14. N. N. Kuznetsov, Accuracy of some approximate methods for computing the weak solutions of a first-order quasi-linear equation, USSR Comput. Math. and Math. Phys. 16 (1976), 105-109. MR 58:3510

15. P. Lions and P. Souganidis, Convergence of MUSCL type methods for scalar conservation laws, C. R. Acad. Sci. Paris. Sér. I Math. 311 (1990), 259-264. MR 91i:65168

16. H. Nessyahu and E. Tadmor, Non-oscillatory central differencing for hyperbolic conservation laws, J. Comput. Phys. 87 (1990), 408-463. MR 91i:65157

17. S. Osher, Riemann solvers, the entropy condition and difference approximations, SIAM J. Numer. Anal. 21 (1984), 217-235. MR 86d:65119

18. 947-961. MR 87b:65147

19. S. Osher and S. Chakravarthy, High resolution schemes and entropy condition, SIAM J. Numer. Anal. 21 (1984), 955-984. MR 86a:65086 
20. S. Osher and E. Tadmor, On the convergence of difference schemes to scalar conservation laws, Math. Comp. 50 (1988), 19-51. MR 89m:65086

21. R. Sanders, On convergence of monotone finite difference schemes for variable spatial differencing, Math. Comp. 40 (1983), 19-36. MR 84a:65075

22. C.-W. Shu, Numerical solutions of conservation laws, Ph.D. thesis, UCLA, 1986.

23. G. Jiang and C.-W. Shu, On a cell entropy inequality for discontinuous Galerkin methods, Math. Comp. 62 (1994), 531-538. MR 94h:65099

24. P. K. Sweby, High resolution schemes using flux limiters for hyperbolic conservation laws, SIAM J. Numer. Anal. 21 (1984), 995-1011. MR 85m:65085

25. E. Tadmor, Convenient total variation diminishing conditions for nonlinear difference schemes, SIAM J. Numer. Anal. 25 (1988), 1002-1014. MR 90b:65173

26. A. Vol'pert, The space BV and quasi-linear equations, Math. USSR Sb. 73 (1967), 255-302. MR 35:7172

27. P. Woodward and P. Colella, The piecewise-parabolic method (PPM) for gas-dynamical simulations, J. Comput. Phys. 54 (1984), 115-173. MR 85e:76004

28. H. Yang, An artificial compression method for ENO schemes: the slope modification method, J. Comput. Phys. 89 (1990), 125-160. MR 91g:65214

29. , Nonlinear wave analysis and convergence of MUSCL schemes, IMA Preprint Series 697, 1990.

Department of Mathematics, Kansas State University, Manhattan, Kansas 66506

E-mail address: hyang@math.ksu.edu 